\title{
L'ISTROROMANZO IN UNA RECENTE PUBBLICAZIONE LINGUISTICA
}

Aggiunte, commenti, rettifiche, risposte alla problematica istroromanza nel volume omaggio a Žarko Muljačić Romania et Slavia Adriatica

1. Il recente volume omaggio a Žarko Muljačić Romania et Slavia Adriatica (Hamburg, Buske Verlag, 1987) riserva, come è naturale, una notevole parte dello spazio ai dialetti chiamati istroromanzi o istrioti: infatti, sui 41 contributi ben 8 concernono l'istroromanzo (in seguito: IR). Vi sono discusse o almeno toccate tutte le questioni della genesi, della storia e della posizione dell'IR nella Romània. Prescindendo ovviamente dal nostro contributo, intendiamo soffermarci sul testo introduttivo di G. Holtus e J. Kramer Streiflichter auf Forschungen zum Dalmatischen und zum Istroromanischen, pp. 43-53 (soprattutto p. 48 sgg.) e sui contributi di J. Kramer (Was sind italienische Mundarten? Bemerkungen zur Klassifikation des «Istroromanischen», pp. 91-100), E. Blasco Ferrer (L'istroromanzo, una lengua-puente. Analisi tipologica e genetica della desinenza di $1^{a}$ persona dell'indicativo presente, pp. 101-113) e G. Ineichen (Bemerkungen zur Stellung des Istriotischen, pp. 115-125). Ci soffermeremo inoltre sul testo di M. Iliescu (Les caractéristiques de la flexion synthétique des verbes réguliers en istro-roman en perspective romane, $\mathrm{pp}$. 365-372), mentre non abbiamo trovato elementi discutibili negli articoli di M. Doria (Note etimologiche al lessico istro-veneto ed istrioto, pp. 255-265) e di G. Holtus (Beiträge zur Lexikographie des Istroromanischen: der «Vocabolario giuliano» von Enrico Rosamani, pp. 525-535).

2. Ciò che sentiamo di dover discutere si divide in alcune sezioni che in parte riflettono l'articolazione della problematica stessa dell'IR, in parte commentano e/o correggono determinate affermazioni nei tre studi citati. La prima sezione è dedicata alla questione della terminologia; la seconda alla discussione della posizione linguistica dell'IR; la terza si sofferma sulla posizione del sottoscritto nella «questione istroromanza» (sit venia verbo); la quarta cerca di tracciare il quadro di alcune importanti caratteristiche dell'IR e della sua divisione interna; la quinta discute l'ipotesi di E. Blasco Ferrer sulla genesi della desinenza $-i$ e sulla definizione dell'IR ivi proposta; la sesta ed ultima, infine, è una breve conclusione. Va da sé che nei limiti del presente testo i molti importanti problemi della linguistica IR possono essere trattati appena per sommi capi. La trattazione esauriente esigerebbe un intero volume.

3. J. Kramer (op. cit., p. 91) afferma che gli studiosi iugoslavi si servono per lo 
più del termine istroromanzo (scr. istroromanski, ted. istroromanisch), creato da Skok nel 1943 e preferito soprattutto da Deanović e dai suoi discepoli. Ma le cose non stanno proprio così. Anzitutto, il Kramer non menziona - e avrebbe dovuto farlo - la curiosa inversione nell'uso terminologico dei due linguisti iugoslavi: mentre Skok nel 1936 parla di istriote (prévénitien) e sette anni dopo di istroroman (1943), Deanović ha esordito col termine istroroman (1952) per ritornare, dopo un periodo di coesistenza dei due termini, alla prima denominazione di Skok (v. Deanović 1954a, 1954b, 1954c, 1955a, 1955b, 1960, 1962, 1965a, 1965b). Del termine istrioto si serve anche $\mathrm{D}$. Cernecca, studioso del dialetto di Valle, il terzo dei tre principali dialetti IR. Non si può dunque dire che gli studiosi iugoslavi utilizzano principalmente il nome istroromanzo, né che questa sia una loro Gepflogenheit (come si legge nel contributo di Ineichen, p. 115). Quanto al sottoscritto, egli preferisce il termine istroromanzo (istroromanski, istroroman) malgrado la sempre possibile confusione con istroromeno (istrorumunjski, istroroumain), dandogli però un significato che evita la petitio principii che $\mathrm{C}$. Tagliavini ha cercato di hineininterpretieren nell'uso terminologico di Skok e del primo Deanović. Per l'autore di queste pagine i termini formati col nome romanzo non pregiudicano necessariamente la posizione attuale dei rispettivi dialetti ma indicano soltanto la dimensione storica, cioè «l'elaborazione» (col noto termine ascoliano) del latino. Inteso così, istroromanzo significa 'romanzo indigeno dell'Istria'; ora, che ci sia stato uno strato latino in Istria che si continua in situ fino ai nostri giorni, è fuori dubbio e fuori di qualsiasi discussione. Nel senso che noi attribuiamo ai termini linguistici in -romanzo si può parlare non solo di istroromanzo ma anche di venetoromanzo, lombardoromanzo, calabroromanzo, aquitanoromanzo, picardoromanzo ecc. ecc. Il problema della posizione di questo istroromanzo nella compagine linguistica istriana, circum-istriana, circum-adriatica e romanza in genere è altra cosa, ma su questo ritorneremo un po' più avanti.

4. Nell'IR noi vediamo dunque la propaggine del romanzo indigeno dell'Istria. G. Ineichen si esprime così: «der Standpunkt von Tekavčić [...] ist allerdings nur dann vertretbar, wenn man das Istriotische (mit der Intuition von Ascoli 1873, 435) als autochthon begreift. Aber alle romanischen Sprachen und Dialekte sind ursprünglich irgendwo im Römischen Imperium autochthon» (p. 122). Ammettiamo che ci sfugge la ragione di queste parole. La prima parte del passo citato è tautologica, dunque inutile: se Tekavčić sostiene l'autoctonia dell'IR in Istria, è ovvio, addirittura lapalissiano, che difendere la sua tesi vuol dire difendere la tesi dell'IR come romanzo autoctono in Istria. La seconda parte lascia perplessi, per non dire altro. Un'altra volta, è lapalissiano che ogni idioma deve essere autoctono in qualche parte (non solo dell'Impero romano ma addirittura della Terra!), ma dal punto di vista delle singole aree - ed è il punto di vista che qui ci interessa-ci possono benissimo essere idiomi indigeni e idiomi importati. Non sarà mica necessario ricordare i risaputi casi del castigliano (autoctono nel Nord della Penisola Iberica, non nel CentroSud) e del còrso (importato dalla Toscana)! Anche l'IR è autoctono in qualche parte (in Istria, naturalmente), ma in Istria ci possono essere anche idiomi importati. Essere scettici di fronte alla distinzione autoctono / importato perché ogni idioma è 
autoctono in un'area, è lo stesso come sentirsi scettici di fronte, ad esempio, alle ricerche statistiche sul movimento della popolazione con l'argomento che ognuno deve per forza essere nato in qualche parte...

5. Si sa da tempo che sulla posizione linguistica dell'IR nella Romània sono state formulate quattro tesi, di cui soltanto due sopravvivono nella linguistica romanza attuale. Le quattro tesi sono esposte in modo succinto da E. Blasco Ferrer (pp. 103-104), ma senza commenti. Oggi non hanno più sostenitori la tesi di A. Ive e Cl. Merlo sull'affinità ladino-istroromanza né la tesi di $M$. Deanović sulla posizione completamente indipendente dell'IR (opinione che non è stata provata). Rimangono dunque la tesi di Skok (a cui si è unito un mezzo secolo fa E. Kranzmayer /1939/) e quella di quasi tutti i linguisti italiani. Quest'ultima vede nei dialetti IR un membro arcaico del gruppo veneto ( $\mathrm{ma}$ in tal caso non può essere logico il termine istriano preveneto, usato ad es. da C. Battisti in Enciclopedia Italiana $19 / 1933 /$, p. 684; secondo Deanović 1955a, p. 58). È errato tuttavia includere fra i sostenitori della tesi di Deanović anche il sottoscritto, come fa E. Blasco (p. 104); infatti, l'autore delle presenti pagine aderisce semmai alla tesi di Skok e di Kranzmayer sull'affinità origi naria, cioè altomedievale, della romanità indigena istriana (da cui nasce l'IR) alla romanità della costa adriatica orientale (che si sviluppa nel dalmatico). La tesi di Skok va beninteso aggiornata, sorretta da prove che siano al corrente della linguistica dei nostri tempi ed appoggiata da esempi sottoposti al necessario vaglio critico. Eliminando tutto ciò che Skok aveva riunito acriticamente, restano pur sempre alcuni argomenti ed esempi validi, su cui abbiamo scritto in precedenza (Tekavčić 1977, nota $20 ; 1979, \S \S 17-18$ ). Oltre a toponimi come Koper e Pićan (le cui basi romanze postulano una $/ \mathrm{p} / \mathrm{risp}$. una $/ \mathrm{t} /$ intervocaliche, e che non possono essere stati imprestati prima del VII o VIII sec.) o Kršikla (con il nesso / $\mathrm{kl} /$ conservato) e appellativi lako 'stagno' (concetto importantissimo in Istria, da sempre povera di sorgenti d'acqua) e skutuler 'cucchiaio grande' (tratto dalla base SCUTELLA), ${ }^{1}$ si può citare anche la differenza tra la caduta della /e/ finale dopo consonanti brevi /l, n, r, s/ e la sua sostituzione con la /o/ dopo consonanti lunghe (geminate) e dopo nessi: -TORE > -dur/-dor, -E(N)SE > -is/-es, contro TURRE > turo/toro, -SSET (3. pers. cong. imperf.) $>$-so, DENTE $>$ dento ecc. V. anche $\S 13$. Questo è un indizio della sopravvivenza relativamente lunga della quantità consonantica e ciò a sua volta potrebbe provare indirettamente anche la non-sonorizzazione delle consonanti sorde brevi intervocaliche $\left(/ t_{1}>d /\right.$ renderebbe possibile $\left./ t \mathrm{t}>\mathrm{t}_{2} /\right)$. E il nome di Latini, che la popolazione croata riserva agli abitanti dei centri urbani di lingua istroromanza? Esso conferma i contatti antichissimi, risalenti ai primi secoli del medioevo, tra Slavi (Croati) e Romani istriani; il suo significato, poi, non entra in nes-

1 Non sappiamo se fra gli esempi di conservazione delle sorde intervocaliche si possa citare anche il verbo spetrà 'purgare da sassi' registrato in Dalla Zonca 1978 e ovviamente derivato dalla base PETRA. Poiché non ne abbiamo trovato altri riscontri, può darsi che si tratti di un italianismo dell'autore. Se al contrario la forma è autentica, è un prezioso esempio di non-sonorizzazione. 
suno dei gruppi semantici stabiliti da Ž. Muljačić (1970) per il morfema latin in serbocroato, e non è, con tutta evidenza, di origine dotta. ${ }^{2}$ Ora, questo nome conserva la / $t$ / intervocalica. E che la voce LATINU, in evoluzione popolare, possa subire la sonorizzazione, là dove questa è un processo indigeno, lo provano gli esiti dolomitico, engadinese e giudeospagnolo (cfr. REW 4927).

6. Il sottoscritto sostiene dunque anche in questa occasione la tesi esposta, e in questo - con le parole di Holtus e Kramer (p. 49) - egli "wird nicht müde» [continuando la medesima immagine: «und warum sollte er müde werden, wenn er von der Richtigkeit dieser These überzeugt ist?»]. E la sostiene senz'alcun motivo politico, contrariamente all'insinuazione (loco ult. cit.) che i linguisti iugoslavi attribuiscono all'IR lo status di linguaggio romanzo autonomo «nicht zuletzt aus politischen Gründen». E che dire di E. Kranzmayer? Aveva anche lui motivi politici?

7. A proposito della tesi sull'affinità istroromanzo-dalmatica c'è una grande differenza tra ciò che si legge in Blasco Ferrer (p. 104) e in Kramer (p. 92). Mentre il primo autore menziona correttamente come sostenitore di questa opinione al primo posto Skok, il Kramer non cita affatto Skok ma si contenta di aggiungere di sfuggita che «fehlte es auch nicht an Stimmen, die auf die Nähe zum Dalmatischen verwiesen», citando in nota soltanto il lavoro di Kranzmayer del 1939. È fuori dubbio che fra i sostenitori della tesi dei contatti istroromanzo-dalmatici il nome di Skok andava assolutamente citato, e precisamente non in forma di «fehlte es auch nicht an Stimmen» ma al primo posto! Non riusciamo a spiegarci l'assenza del nome del principale propugnatore di questa tesi nello studio di Kramer.

8. Che cosa sono dunque per noi i dialetti IR e che posizione nella Romània attribuiamo loro? Ripetiamo in breve quello che si è già detto, e cerchiamo di aggiungervi alcune altre idee che riteniamo importanti.

8.1 In aree come l'Istria, territori di contatti e di stratificazioni, esposti da secoli «ai quattro venti», la classificazione che voglia essere realistica non può essere statica ma dinamica; deve tener conto, cioè, delle possibilità di spostamento di un idioma, del suo allontanamento da un centro e avvicinamento ad un altro. Per non andare in cerca di esempi lontani, citiamo la storia dei dialetti italiani settentrionali, che dall'orientamento galloromanzo altomedievale si sono orientati il sud, verso la Toscana. Crediamo che uno spostamento analogo si sia verificato anche nei dialetti IR lungo i dodici secoli circa che separano l'inizio del periodo romanzo dall'epoca delle prime attestazioni dell'IR.

2 Per l'Istria altomedievale può valere $a$ fortiori ciò che V. Foretić $(1987$, p. 502) constata a proposito del nome Latini a Zadar e a Split. A differenza di Dubrovnik, dove questo nome può significare 'cattolici', a Zadar e a Split «gab es im Mittelalter überhaupt keine Einwohner orthodoxen Glaubens. Deshalb wurde der Begriff Latini hier in ethnischer Hinsicht verwendet, um die Romanen (die Latini) von den Kroaten abzusetzen». 
8.2 Che l'IR sia un'entità dialettale italiana (veneta) nella sua fase moderna, è pacifico e ammesso persino da Deanović, e anche noi possiamo sottoscrivere le sue parole «Siamo, beninteso, tutti d'accordo che l'odierno istrioto sia da considerare come ormai una varietà del veneto» $(1962$, p. 378$)$. Sforzarsi a provare l'italianità dell'IR moderno vuol dire dunque sfondare una porta aperta. L'italianizzazione, più precisamente la venetizzazione pressoché completa dell'IR è un fatto, ed è appunto in questo che si esplica lo spostamento di cui poco prima abbiamo parlato, dunque anche la necessità di una classificazione dinamica. L'attrazione nell'orbita veneta si osserva anche altrove, ad esempio nella convergenza progressiva venetoragusea, studiata da Muljačić. Si può affermare senza paura di errare che il raguseo, se fosse vivo oggi, sarebbe altrettanto venetizzato come l'IR; eppure, all'origine il raguseo, parte del dalmatico, era naturalmente «un'elaborazione del latino propria e indigena di Ragusa», non certamente un dialetto italiano (veneto)! Ma quello che importa soprattutto è la ricostruzione indiretta della prima fase dell'IR, quella altomedievale. Per citare di nuovo Deanović (loco ult. cit.): «ma quello che ci interessa di più non è il suo stato attuale, bensì la genesi e la struttura alle sue origini medievali». Si ritorna, insomma, alla tesi di Skok.

8.3 Va tenuto presente anche un altro momento (cfr. per un accenno in questo senso Tekavčić 1982, p. 277). Dato che l'IR ci è attestato soltanto nella sua ultima fase (dal 1835 ad oggi), che è un decimo circa del suo periodo di vita, mentre i toponimi e i relitti lessicali nei dialetti croati istriani sono molto più antichi, risulta che non tutti i livelli dell'analisi linguistica avranno la medesima importanza ai fini della ricostruzione e della classificazione. La morfologia e soprattutto la sintassi, essendo per forza delle cose desumibili solo dai testi, si situano ad una tappa notevolmente più recente della fonologia e del lessico.

8.4 Ha ragione Ineichen quando constata che «heutzutage weichen hier [nel problema della posizione dell'IR] viele Autoren auf die Gesichtspunkte der Soziolinguistik aus» (p. 121). Sebbene fra quelli che «occasionalmente» procedono così si trovi citato anche il sottoscritto, questi è del parere che bisogna distinguere il punto di vista linguistico da quello sociolinguistico, in IR come in qualsiasi altro dominio linguistico. L'ottica sociolingiustica è valida per la distinzione lingua/dialetto, non è invece rilevante per la classificazione puramente linguistica. Kramer adatta ai dialetti italiani lo schema di J. Goossens (per i dialetti tedeschi) basato sul concetto di «Überdachung» e la definizione di W. Th. Elwert la cui base sono le «Kultursprachen» (p. 98), ma nel caso del còrso non lo considera come dialetto francese (malgrado la «Überdachung») bensì come dialetto italiano, perché «das Korsische ist jedoch leichter ins Diasystem des Italienischen als in das einer anderen romanischen Sprache, etwa des Französischen, einzuordnen» (loco ult. cit.), Qui si opera evidentemente con criteri linguistici, non sociolinguistici né $A$ usbau-comparatistici; allora, perché nel caso dell'IR non si dovrebbe applicare lo stesso procedimento? L'IR moderno è senza dubbio tipologicamente italiano (veneto), ma l'IR altomedievale presenta un quadro ben diverso. Nell'IR, che sociolinguisticamente è sempre stato ed è tuttora dialetto, non lingua, non ci è di alcun aiuto la sociolinguistica né la Ausbau- 
komparatistik, perché i due problemi di fondo sono: 1) la ricostruzione della prima facies (altomedievale) di questo idioma, 2) il suo posto nella Romània.

8.5 Per essere oggettivi e completi, dobbiamo dissentire in un punto anche dalle parole or ora citate di Deanović: se, cioè, il problema principale è la ricostruzione della prima fase dell'IR, non si deve per niente intendere che le fasi posteriori e la fase moderna non ci interessino. Al contrario, la descrizione e l'analisi della fase moderna è la base indispensabile, il punto di partenza logico ed obbligatorio, per la ricostruzione. È dunque normale che, secondo Ineichen (p. 120), «Tekavčić eine jüngere Phase des Dialektes beschreibt».

\section{IV}

9. Occupiamoci adesso di alcuni tratti importanti dell'IR. Chiunque conosce la complessità di questo gruppo dialettale dovrà dissentire da Kramer, il quale afferma che l'IR «man trotz der natürlich von Ort zu Ort feststellbaren kleineren Unterschiede für die hier interessierende Frage [la posizione dell'IR] ruhig als Einheit behandeln kann» (p. 93). Dal passo non risulta quali sono le differenze a cui Kramer allude, ma una cosa è certa: la differenza fondamentale o quanto meno una delle fondamentali è la presenza o meno dei dittonghi discendenti /ey, ow/ in IR. Nei dialetti rovignese (RO), dignanese (DI) e fasanese (FA) i dittonghi citati ci sono, nel vallese (VA), gallesanese (GA) e sissanese (SI), nonché nell'estinto polesano (PO) non ci sono. Ora, questa non è una differenza minore o lieve («kleinerer Unterschied») ma un'isoglossa che entra proprio nel fondo dell'IR, nella sua genesi e pertanto anche nel problema della sua posizione nella Romània. Questi dittonghi sono da sempre al centro degli studi IR, dai tempi di Ive fino al recente contributo di F. Ursini (1983). Proprio perché sono sentiti come caratteristica importante, tratto tipico, abbonda il loro uso ipercorretto (ipercaratterizzante) sia nel Vocabolario dignanese-italiano di G. A. Dalla Zonca (1978) che nei testi rovignesi dell'antologia Istria Nobilissima (v. Tekavčić 1986 e 1987). Un'altra differenza, non meno importante (su cui avremo occasione di ritornare fra poco), è a livello morfologico: in RO e FA si ha nella 1 persona del presente indicativo la desinenza latina conservata (in RO - $o$, in FA - $u$, in seguito alla tendenza generale verso la chiusura della /o/ finale in $/ \mathrm{u} /$, cfr. Ive 1900 , $\S 39$, p. 141), negli altri quattro dialetti (VA, DI, GA, SI) appare nella stessa persona la desinenza $-i$, il che porta all'omofonia tra la 1 e la 2 persona (DI: kanti 'canto' e 'canti', veñdi 'vendo' e 'vendi', dormi 'dormo' e 'dormi'; analogamente negli altri tre dialetti del gruppo). Una terza isoglossa nel dominio IR è la presenza o meno dei dittonghi ascendenti /ye, wo/: essi sono assai frequenti e netti in RO e GA (e precisamente tanto i dittonghi primari, risalenti ai fonemi /ę, o/, quanto i secondari, provenienti dai dittonghi /ay/e /aw/), mentre non si trovano nei dialetti VA e DI. Differenze ugualmente notevoli ci sono nel consonantismo: per 'chiesa', dal. lat. ECCLESIA, Ive registra in alcuni dialetti IR due, talvolta persino tre forme: nel DI čiza, ğeza e jeiza, nel GA čiza e jeiza, nel FA čeza e gezia (Ive 1900, p. 4, nota 2). Abbiamo dunque persino cinque esiti di ECCLESIA: čeza, čiza, ğeza, gezia, jeiza. Vi si notano due esiti di /ē/. (/i/ e /e/) e ben tre esiti del nesso $/ \mathrm{kl} /(/ \check{c} /, / \grave{\mathrm{g}} /, / \mathrm{j} /)$. 亡̀ 
chiaro che queste forme non possono appartenere ad un solo strato linguistico. Un'altra isoglossa morfologica: il DI, a differenza degli altri dialetti, riduce la desinenza-emo (< -EMUS) della 4. persona del presente indicativo a -en (kantén, vendén, durmén), negli altri dialetti la vocale finale /o/ (FA /u/) si mantiene. E si potrebbero citare diverse altre differenze fra i singoli dialetti IR. Abbiamo dunque il diritto di parlare di un IR? V. Per questo Tekavčić 1972-73, 1977, 1979. Se da un lato è ovvia la necessità di distinguere alcuni strati (che si riflettono nei citati esiti duplici o triplici), dall'altro lato è altrettanto evidente che non abbiamo alcun diritto di considerare ad esempio i dialetti emiliano-romagnoli come non-italiani perché presentano i dittonghi discendenti e i dialetti veneti come italiani perché non ne conoscono. Per risolvere questi problemi occorre stabilire criteri tipologici quantificabili, necessari e sufficienti per la classificazione delle singole varietà IR, e soprattutto raccogliere quanto più materiale dialettale.

10. Ci resta da commentare il tentativo di E. Blasco Ferrer di render conto della desinenza $-i$ nella 1 persona del presente indicativo in quattro dialetti IR (VA, DI, GA, SI). Questo fenomeno serve all'autore da base per attribuire all'IR la posizione di «lengua-puente» tra la Romània meridionale conservativa e la Romània centrale innovativa (p. 111). La tesi si può riassumere così:

Partendo da una breve rassegna tipologica e dal quadro generale delle desinenze per la 1 persona in IR e altrove nella Romània, l'autore passa poi ad un prospetto sinottico delle forme IR, che mostrano tre omofonie: 1) RO: $1=3$; 2) PO, PI [piranese], FA: $2=3 ; 3$ ) VA, DI, GA, SI: $1=2$. Nel nucleo del suo contributo Blasco Ferrer cerca di trovare una spiegazione «endogena», ricavata cioè dal solo paradigma del presente, e crede di trovarla nella sostituzione della desinenza - $o$ per la 1 persona con $-i$. Le forme vendo, dormo ecc. vengono sostituite dalle forme in $-i$ (vendi, dormi) per evitare l'omofonia tra la 1 e la 3 persona, causata dalla sostituzione della /e/finale con la /o/ (per cui VENDIT > vende, poi vendo diventa omofono con VENDO > vendo; $\mathrm{v}$. anche av.). La sostituzione $-e>-o$ è definita un passaggio «davvero inaudito nella Romània» (p. 109). Le due sostituzioni fanno sì che un'omofonia $(1=3$ pers. $)$ venga sostituita da un'altra $(1=2$ pers. $)$, ma questa ultima è secondo l'autore meno pericolosa di quella, perché il contesto e la presenza degli interlocutori rimediano al pericolo dato dal sincretismo formale. Quanto al RO, che non si inquadra nell'ipotesi dell'autore perché presenta la desinenza -o tanto nella 1 quanto nella 3 persona, Blasco Ferrer cita esempi tratti dai Canti popolari istriani di Ive (1877) come VOCE > buse, DICIT > deîse (p. 107, nota 13) e conclude che la sostituzione $-e>-o$ è in RO un cambiamento «tardo e poco energico» (p. 110). Egli cita inoltre le forme per 'so', 'posso', 'voglio' e 'vengo' nei dialetti PO, PI e RO (loco ult. cit.) e ritiene autoctona in IR la forma so 'so', mentre se 'idem' sarebbe un prestito dal veneto. La conclusione (posizione di «lengua-puente» dell'IR) è stata già citata. 
Il contributo dell'autore contiene una serie di punti discutibili, anche errati, che mettono in dubbio l'intero ragionamento e la conclusione che ne dovrebbe risultare.

11. L'autore rimprovera al sottoscritto di trascurare i dialetti PI e PO (p. 107) i quali invece nella sua tesi occupano un posto importante, quasi centrale. Ora, il PI descritto da Ive non era un dialetto IR ma veneto (cfr. Cortelazzo 1972), isolato sia arealmente che tipologicamente dall'IR e da Ive erroneamente incluso tra i suoi dialetti «ladino-veneti». Se si include nell'analisi il PI, si devono logicamente prendere in considerazione anche tutti gli altri dialetti veneti dell'Istria. Quanto al PO, esso non esiste più e già ai tempi di Ive era nettamente diverso dai sei dialetti IR conservati fino ad oggi (RO, VA, DI, FA, GA, SI): si vedano, tanto per citare un esempio, i paradigmi dei verbi irregolari ( $\S 171-182)$. I dialetti PI e PO non possono dunque essere trattati allo stesso modo degli altri sei dialetti e soprattutto non è metodologicamente corretto costruire spiegazioni che, in base ad essi, siano valevoli per tutto l'IR.

12. La constatazione che il passaggio (noi preferiamo parlare di sostituzione) - $e$ $>-o$ sia «inaudito nella Romània» è certamente esagerata: infatti, oltre all'IR, al veglioto e al raguseo (citati dall'autore a p. 109), presentano lo stesso fenomeno anche i dialetti veneti ad es. di Lio Mazor e di Verona, il dialetto della Brianza, la colonia gallo-italiana di Nicosia in Sicilia e la Toscana nord-occidentale (v. Rohlfs 1966, $\S 143$, con abbondanti esempi, di cui diversi sono identici a quelli IR).

13. Non è giustificato nemmeno l'atteggiamento minimizzante dell'autore a proposito della sostituzione $-e>-o$ in RO. Il fenomeno non vi si può assolutamente qualificare di «tardo e poco energico» (formulazione di per sé alquanto strana), perché esso ha in RO esattamente la stessa diffusione che ad es. in DI. Ecco una scelta di esempi, con forme specificamente dignanesi aggiunte tra parentesi:

a) sostantivi: BUTTE $>$ buto, CARNE $>$ karno, CLAVE $>$ čavo, DENTE $>$ dento, FULMINE $>$ fòulmino, GENTE $>$ zento, NOCTE $>$ nuoto (noto), $\mathrm{PISCE}$ $>$ piso, TÜRRE $>$ turo ecc.

b) aggettivi (al masch. sing.): -ANTE/-ENTE > -anto/-ento (acc. a -ante/ente), DULCE $>$ dulso, FORTE $>$ fuorto (forto), GRANDE $>$ grando, VIR(I)DE $>$ virdo ecc. (Il femm. sing. esce in $-a$, essendo gli aggettivi inseriti nella classe sano, - a.)

c) avverbi: -MENTE $>$-mento o-mentro, $\mathrm{AD}+\mathrm{RADENTE}>$ arento (e arente), SEMPER > sempro ecc.

d) la 3 persona dell'indicativo presente: BATT(U)IT $>$ bato, BIBIT $>$ bivo, CURRIT > kuro, LEGIT > lezo $\left(l \varepsilon z o^{3}\right)$, MITTIT > meto (meto), VENDIT > vendo, VIDET $>$ vido ecc.

3 Con $\varepsilon$ trascriviamo la vocale anteriore di notevole apertura, che è fonema in DI, come provano le coppie /leto/ 'partic. di leggere' /ẹto/'mobile', /mєio/ 'meglio' /mẹio/'mio' ecc. 
e) la 3 persona del congiuntivo imperfetto: in RO kantiso, vendiso, durmiso, in DI: kantaso, vendiso, durmeiso ecc.

f) la 3 persona del condizionale: kantaravo, vendaravo, durmiravo, con-avo < HABUIT (cfr. in Italia Rohlfs 1968, § 597).

Si veda anche Ive $1900, \S 29$ delle singole sezioni dialettali e Deanović 1954a (non citato da Blasco Ferrer), p. 17. Tutti i tipi di esempi si trovano anche nei testi rovignesi attuali pubblicati in Istria Nobilissima. Va sottolineato in particolar modo che in RO si trova talvolta -o là dove noi a Dignano abbiamo raccolto forme con -e: a puséibalo 'possibile' (Ive 1900, pp. 176-178) e inpuséibalo 'impossibile' (Ive 1900, p. 65) fa riscontro nel DI (im)pusèybile.

Poiché dunque la sostituzione $-e>-o$ assume in RO esattamente le stesse dimensioni che in DI, VA ecc., sorge il problema principale: se $-e>-o$ ha provocato l'introduzione della desinenza - $i$ per - $o$ nella 1 persona in VA, DI, GA e SI, perché lo stesso non è avvenuto in RO? Perché qui l'omofonia della 1 e 3 persona è tollerata?

14. Si è già detto che per il RO Blasco Ferrer si serve di esempi desunti dai Canti popolari istriani di Ive del 1877. Ma egli trascura il fatto che proprio nei canti popolari ricorrono numerosi influssi veneti e/o italiani. Per convincersene basta citare alcuni esempi desunti dalle poesie riprodotte in Deanović 1954a, pp. 73-76 (con la forma genuina da noi aggiunta fra parentesi): ğuvene 'giovane' (zuvana, masch. $z u$ vano); vuria 'vorrei' (vularavi); benedita 'benedetta' (banadita); bevarié 'berrai' (bivarié); rekurdarié 'ricorderai' (rakurdarie); poi gli infiniti in -re per necessità metriche: puğare, maridàre, mazenare, tsapare, (puğà, maridà, mazanà, sapà) ecc. Anche nei volumi di Istria Nobilissima si leggono strofe con infiniti come andare, vinei$r e$, piassire, guantare 'vantare' (vol. VII, p. 161) per le forme genuine zei (< IRE), viñei, piàzi (< *PLÁCERE per PLACÉRE), guantà. È fuori dubbio che i canti popolari in Istria non sono la migliore fonte per la lingua veramente popolare, indigena.

15. Alla spiegazione proposta da Blasco Ferrer si può obiettare anche che la provenienza della desinenza $-i$, destinata a rimediare all'omofonia di vendo $(<$ VENDO) e vendo (< VENDIT), non è spiegata. Da dove salta fuori la $-i$ ? Tali sostituzioni non avvengono da un giorno all'altro, in modo teleologico (thései, si sarebbe tentati di dire). Affinché al posto di $-o$ si introduca $-i$ bisogna supporre un periodo di coesistenza di - o e - $i$ (e naturalmente spiegare la provenienza di $-i$ ).

16. Contrariamente all'opinione di Blasco Ferrer, l'omofonia tra la 1 e 2 persona non è meno pericolosa di quella tra la 1 e 3 persona; viceversa, quest'ultima non arreca più danno al funzionamento di quella. L'autore trascura il fatto che in IR le forme verbali personali sono sempre accompagnate da sostituenti clitici in funzione di soggetto (cfr. Iliescu, p. 366, nota 4: «Les distinctions des personnes dans l'acte de communication sont sauvées par les pronoms sujet non accentués»). Anzi, i sostituenti clitici sono talmente frequenti che in tutti i materiali IR abbondano casi 
come Al paron al ge dei a la murieda 'Il padrone dice alla ragazza', col soggetto espresso dal sostantivo e dal sostituente clitico. I clitici in IR rimediano a qualsiasi omofonia delle forme verbali.

17. Alla luce di quanto visto poco fa sulla diffusione di $-e>-o$ in Italia, non crediamo che c'entri l'influsso dello slavo, il che si legge nella nota $20 .{ }^{4}$ Per quanto poi riguarda la sostituzione della vocale affievolita in «suono centro-posteriore alto» (loco ult. cit.), un'ipotesi del tutto analoga è stata da noi formulata nella nostra comunicazione al XIV Congresso di Linguistica Romanza (Napoli 1974), non citata dall'autore (Tekavčić 1976). La nostra proposta parte dalla differenza tra la caduta

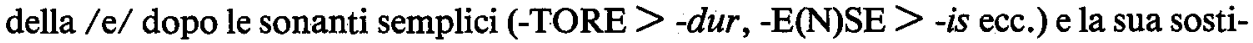
tuzione con la /o/ dopo le sonanti lunghe (geminate) e dopo nessi (TURRE $>$ turo, -SSET nella 3 pers. cong. imperf. $>$-so, DENTE > dento); cfr. sopra, § 5. Questa differenza prova che dopo consonanti lunghe e nessi la /e/ non è caduta del tutto (se così fosse, i due tipi di contesti fonetici si sarebbero identificati) ma si è ridotta a $/ \partial /$, fonema vicino alla $/ \mathrm{o} /$ per cui più tardi, sotto l'influsso veneto, è stato sostituito appunto dalla $/ \mathrm{o} /$. Se si fosse avuto il dileguo totale della $/ \mathrm{e} /$, il modello veneto con la sua /e/ conservata avrebbe determinato la restituzione di /e/ anche in IR. La trafila è dunque TURRE $>$ *turra $>$ turo, DENTE $>*$ denta $>$ dento.

18. A differenza dell'autore non vediamo in se 'so' un'importazione veneta. Se so è la forma propria del PO e del PI, mentre se vive negli altri dialetti (per la precisione: in RO e GA siè), e se sappiamo che proprio il PO e il PI si scostano dall'IR presentando numerosi paralleli col veneto, è ovvio che se (siè) dovrebbe essere IR genuino, so invece un importo dal veneto, o dall'italiano standard. Del resto se(siè) è strettamente simmetrico a iè 'ho' in RO, VA, DI, GA e SI, il FA ha caratteristicamente iè acc. al moderno go, mentre il PO e il PI conoscono soltanto le forme $o$, go, ga ecc. (Ive 1900, $§ 173$ dei singoli dialetti).

19. E. Blasco Ferrer definisce la nostra speigazione (che parte da una presuppo-

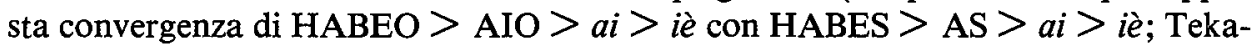
včić 1975) come intraparadigmatica (p. 108) e le contrappone la sua, definita endogena (p. 109), cioè «reperibile all'interno dello stesso paradigma del presente». Ma anche la nostra spiegazione è endogena in questo senso, perché non esce dal presente. D'altra parte, però, l'autore ammette influssi esogeni [là dove, a quanto pare, non ci sono fattori endogeni reperibili], ad es. in pói, puói 'posso' (p. 110). Quale influsso? Non certamente veneto. E perché proprio in questa forma? Come si può giustificare il prestito di una sola forma di un paradigma? E si tenga presente che per la forma vuói 'voglio', in tutto parallela a puói, si suppone la caduta della /o/ per tenere distinta la forma affermativa da quella interrogativa, col sostituente affisso

4 Le semivocali slave si perdono in posizione finale senza traccia, e certamente troppo presto per poter esercitare un influsso sull'IR, come vuole E. Blasco Ferrer. Cfr. ad es. Hamm 1958, p. 80; Iv̌̌ić 1970, soprattutto p. 105. D. Malić ha studiato recentemente la lingua della cosiddetta Carta di Povlja (Povaljska listina) del 1250 e ha constatato che la semivocale vi si scrive ancora, «benché in fine di parola e nella posizione debole essa sia da tempo scomparsa nella lingua viva» (Malić 1987, p. 89). 
(vuóio? 'voglio io?'). Perché qui non c'entrano fattori esogeni? Perché due processi diversi per le forme in tutto parallele?

20. Si aggiunga che l'effetto del preteso bisogno di distinguere la 1 dalla 3 persona mediante l'introduzione della desinenza $-i$ si trova anche nei verbi irregolari, dove una tale omofonia non c'era: anche con -o le forme deigo 'dico', dago 'do', fago 'faccio', vieño 'vengo' ecc. si distinguono bene dalle relative 3 persone (dei o deis, $d a, f a$, ven). Eppure, la desinenza $-i$ si trova (sempre nei quattro dialetti: VA, DI, $\mathrm{GA}, \mathrm{SI})$ anche in questi verbi.

21. Infine, Blasco Ferrer non considera l'importante fatto che là dove la desinenza $-i$ si trova nella 1 persona del presente, la stessa desinenza ritorna nella 1 persona dell'imperfetto (ad es. in DI: kantàvi 'cantavo' e 'cantavi', vendivi 'vendevo' e 'vendevi', durmèivi 'dormivo' e 'dormivi'). I due paradigmi sono evidentemente collegati, ma sull'imperfetto l'autore (proprio per la sua spiegazione «endogena») non dice nulla. In questo caso si tratta di un'analogia interparadigmatica: qualunque sia l'origine della $-i$ nel presente, quella dell'imperfetto deve esserne un'estensione analogica secondaria.

22. Le critiche esposte rendono a nostro avviso insostenibile tutta l'ipotesi di E. Blasco Ferrer. Se sia esatta la nostra spiegazione resta soggetto a discussioni ulteriori; e non è escluso che ci sia una terza spiegazione, differente da ambedue e preferibile ad esse. Ad ogni modo, crediamo che la nostra ricostruzione presenti meno punti deboli di quella di Blasco Ferrer.

23. Se è così, viene a cadere anche la definizione dell'IR come «lengua-puente» tra la Romània conservativa meridionale e la Romània innovativa centrale. Prima di tutto: un solo criterio, una sola isoglossa non possono bastare mai per assegnare ad un idioma un certo status. Ma, anche ammesso che possiamo procedere così, applicando conseguentemente il criterio adottato da Blasco Ferrer, dovremmo attribuire alla Romània conservativa il dialetto veneto e tutti gli altri dialetti italiani settentrionali che conservano la desinenza latina $-O$, mentre apparterrebbe alla Romània innovativa ad esempio il milanese e gli altri dialetti che presentano la desinenza $-i$. Ma nemmeno questo è ancora tutto. Come mostra il RO, il criterio stesso delle desinenze $-o$ e $-i$ perde la sua validità. Nei dialetti RO e DI la sostituzione $-e>-o$, ripetiamolo un'altra volta, ha esattamente le stesse dimensioni, eppure il RO conserva la desinenza latina, il DI (assieme agli altri tre dialetti) la sostituisce con $-i$. Dobbiamo dunque assegnare il RO (ed il FA, con $/ \mathrm{o}>\mathrm{u} /$ in posizione finale) alla Romània conservativa, il DI (con il VA, il GA, ed il SI) alla Romània innovativa? Ovviamente, questo sarebbe assurdo. Divisioni dialettali intra-istroromanze ci sono, beninteso, e la desinenza -o risp. $-i$ è soltanto una di esse. Ognuna di queste isoglosse fornirebbe un quadro per poco che sia diverso; allora, perché appunto la desinenza della 1 persona del presente indicativo dovrebbe servire per classificazioni di così vasta portata?

24. Infine, aggiungiamo qualche parola sui vari sbagli fattografici nel contri- 
butto di M. Iliescu. P. 365: il RO è appena più settentrionale del VA, il PI non fa parte del gruppo IR, ed il PO non viene menzionato affatto; p. 366: se si considera tutto l'IR, l'infisso del presente è - $e$ - o- $i$ - nella I classe, $-i s$ - o -eis- nella IV classe; p. 367: l'imperativo negativo singolare è possibile anche senza $a$ (dunque: $n u$ sta favalà 'non parlare'); p. 368: la desinenza della 3 persona del futuro è -ò, non -à (in RO e GA: -uò); ib.: non ci risulta chiaro in che senso il segmento - $a v$ - del condizionale sia "variante de l'imparfait»: dal punto di vista sincronico il contatto morfematico tra i due paradigmi non si avverte, diacronicamente guardando - $a v$ - risale al perfetto di HABERE; p. 369: la vocale del gerundio precedente il segmento -ndo non è -á-, - $i-$-, $-\dot{u}$ - ma - $a$ - (I classe), -e-, anche $i$ (altrove) =-ando, -endo, -indo; ib.: il SM (suffisso modale) del participio è $-d$ - anche nel maschile plurale (purtadi) come nel femminile (purtada-purtade), mentre solo nel maschile singolare è al grado zero (purtà); p. 370: nella Cisalpina si integra beninteso l'IR odierno; ora, quello che importa soprattutto nella linguistica IR, è lo status della prima fase dell'idioma; p. 371: i participi in -é-/-éd-non esistono in IR.

25. Qualche altra correzione di natura piuttosto tecnica (in Holtus-Kramer e Iliescu): a p. 44 Montovun (due volte) va corretto in Motovun; a p. 51 andrebbe citato Tekavčić 1975 per la morfologia di tutto il verbo IR; ib.: Doria 1974 manca nella bibliografia e Tekavčić 1976 va precisato, essendoci due titoli in quest'anno; a p. 53 correggere (in Tekavčić 1976) romanistike in onomastike; a p. 365 Tekavčić 1967 va precisato, dato che anche qui due titoli recano lo stesso anno.

VI

26. In conclusione, ecco quanto ci pare di poter dire sulla trattazione dell'IR nel volume omaggio Romania et Slavia Adriatica. L'IR vi occupa un posto notevole e, con tutte le esitazioni dei singoli autori, viene riconosciuto senz'altro come un membro a sé di quella che si può definire Istria romanza. Si ha una certa impressione che gli autori non osino accogliere apertamente la tesi iugoslava, mentre d'altra parte si rendono conto di non poter includere l'IR nel gruppo dialettale veneto tout court. Allora, come constata bene Ineichen, si spostano sul terreno della sociolinguistica. Ma quest'approccio non risolve il problema di fondo, che è la ricostruzione della genesi e del posto dell'IR nella sua prima fase, nonché le tappe ulteriori della sua storia. Questo resta dunque anche dopo il nostro volume omaggio un desiderato; anzi non un ma il desiderato della linguistica IR; il compito che attende i romanisti di domani.

\section{OPERE CITATE}

Cortelazzo 1972: M. Cortelazzo, Tracce dell'antico dialetto veneto di Pirano, «Linguistica» 12 , pp. $31-40$.

Dalla Zonca 1978: G. A. Dalla Zonca, Vocabolario dignanese-italiano, a cura di Miho Debeljuh, Trieste. 
Deanović 1952: M. Deanović, Remarques sur le système phonologique de l'istroroman, «Bulletin de la Société de Linguistique de Paris» 48, pp. 79-83.

Deanović 1954a: M. Deanović, Avviamento allo studio del dialetto di Rovigno d'Istria, Zagreb.

Deanović 1954b: M. Deanović, Ricerche sull'istroromanzo, in: Atti del II Congres- so internazionale dei linguisti, Milano, pp. 61-72.

Deanović 1954c: M. Deanović, Voci slave nell'istrioto, «Ricerche slavistiche» III, pp. 51-68.

Deanović 1955a: M. Deanović, Istroromanske studije, «Rad» Jugoslavenske akademije znanosti i umjetnosti 303, pp. 51-118.

Deanović 1955b: M. Deanović, Nomi di piante nell'istrioto, «Archivio Glottologico Italiano» 39, pp. 187-205.

Deanović 1960: M. Deanović, Sull'istrioto, in: Atti dell'VIII Congresso internazionale di studi romanzi II, pp. 505-513.

Deanović 1962: M. Deanović, Tracce dell'istrioto nell'antica toponomastica dell'Istria, in: Atti e Memorie del VII Congresso internazionale di scienze onomastiche I, pp. 377-384.

Deanović 1965a: M. Deanović, Ugolino in una versione istriota di Rovigno, «Studia Romanica et Anglica Zagrabiensia» (SRAZ) 19-20, pp. 39-56.

Deanović 1965b: M. Deanović, Dal lessico istrioto, in: Omagiu lui Alexandru Rosetti la 70 de ani, Bucureşti, pp. 157-161.

Foretić 1987: V. Foretić, Zur Geschichte der Romanen Dalmatiens im Mittelalter, in: Romania et Slavia Adriatica, Hamburg, pp. 483-503.

Hamm 1958: J. Hamm, Staroslavenska gramatika, Zagreb.

Istria Nobilissima: Antologia delle opere premiate ai Concorsi d'arte e di cultura Istria Nobilissima, Trieste: vol. I 1968 - vol. XVIII 1985.

Ive 1900: A. Ive, I dialetti ladino-veneti dell'Istria, Strasbourg.

Ivšić 1970: S. Ivšić, Slavenska poredbena gramatika, Zagreb.

Kranzmayer 1939: E. Kranzmayer, Frühromanische Mundarten zwischen Donau und Adria in deutschen und slavischen Ortsnamen, «Zeitschrift für Namenforschung» XV, pp. 193-224.

Malić 1987: D. Malić, »Staro« $i$ »novo« $u$ jeziku Povaljske listine, in: Obljetnica Povaljske listine i Praga 1184-1984, Brački zbornik XV, Supetar, pp. 84-101.

Muljačić 1970: Ž. Muljačić, Semantička analiza morfema /latin/, «Onomastica Jugoslavica» 2, pp. 132-142.

Rohlfs 1966-1968: G. Rohlfs, Grammatica storica della lingua italiana e dei suoi dialetti, Torino: Fonetica 1966, Morfologia 1968.

Tekavčić 1972-73: P. Tekavčić, Il comune e lo specifico nel dominio istroromanzo, «SRAZ» 33-36, pp. 639-678.

Tekavčić 1975: P. Tekavčić, Caratteristiche e problemi del verbo istroromanzo, «SRAZ» 39, pp. 55-105.

Tekavčić 1976: P. Tekavčić, Interferenze linguistiche istroromanzo - venete: sulle vocali finali nell'istroromanzo, in: Atti del XIV Congresso di Linguistica e Filologia Romanza (Napoli 1974); II, pp. 447-467. 
Tekavčić 1977: P. Tekavčić, Problemi teorici e metodologici nella ricostruzione dell'istroromanzo, «SRAZ» 43, pp. 35-54.

Tekavčić 1979: P. Tekavčić, Il posto dell'istroromanzo nella Romània Circumadriatica, «SRAZ» XXIV, 1-2, pp. 21-46.

Tekavčić 1982: P. Tekavčić, L'importanza e l'interesse degli studi istroromanzi per la linguistica romanza e generale, «Revue de Linguistique Romane» (RLiR) 46, pp. 271-298.

Tekavčić 1986: P. Tekavčić, Lessicografia istroromanza ieri, oggi, domani, «RLiR» 50, pp. 5-35.

Tekavčić 1987: P. Tekavčić, Le convergenze e le divergenze fonetiche nell'istroromanzo (soprattutto rovignese) ed $i$ loro riflessi nei test $i, « \mathrm{RLiR} » 51, \mathrm{pp} .331$ -350 .

Ursini 1983: F. Ursini, I «dittonghi discendenti» nell'istrioto di Rovigno: un problema fonetico, in: Scritti linguistici in onore di Giovan Battista Pellegrini II, Pisa, pp. 1217-1225.

\section{Sažetak}

\section{ISTROROMANSKI GOVORI U JEDNOJ NEDAVNO IZAŠLOJ LINGVISTIČKOJ PUBLIKACIJI}

Autor ovoga priloga osvrće se na neke (od osam) radova koji u zborniku Romania et Slavia Adriati$c a$ (u čast Žarku Muljačiću, Hamburg 1987) obrađuju ili dodiruju istroromansku (IR) problematiku. U prvom odsječku diskutira teminološka pitanja; drugi se bavi poznatim tezama o položaju IR govorâ u romanskom svijetu; treći izlaže autorove poglede na definiciju i mjesto IR govorâ; četvrtom su tema glavne razlike među pojedinim IR govorima i pitanje jedinstva IR dijalekata; peti komentira i pobija jedno tumačenje (autor E. Blasco Ferrer) nastavka - $i$ u 1 . licu prezenta u većini IR govorâ i iz toga izvedeni zaključak o položaju IR u Romaniji, a zatim ispravlja neke manje pogreške u drugim prilozima; šesti i zadnji odsječak donosi opći sud i smjernice budućih istraživanja. Glavni je problem IR lingvistike rekonstrukcija povijesti tih govora s posebnim težištem na njihovom položaju u romanskom svijetu u najstarijoj, tj. ranosrednjovjekovnoj fazi. Toj problematici ne pridonose ni radovi u spomenutom zborniku, pa ona ostaje i nadalje najvažniji zadatak IR grane romanske lingvistike. 